\title{
Surface Imaging of Surface Non-linear Processes
}

\author{
Harm Hinrich RoTERMUND \\ Department of Physics and Atmospheric Science, Dalhousie University, Halifax, Canada
}

(Received April 9, 2008)

\begin{abstract}
Simple surface reactions like the CO-oxidation on single crystal Pt surfaces show a rich variety of pattern formation under specific reaction parameters. To visualize those patterns we have conceived several unique imaging methods starting around 1990. The interaction of a multitude of micrometer scale concentration waves and fronts on the surface complicate our understanding the underlying mechanisms for such patterns. Experiments with modified catalytic activity using stationary, inactive boundaries have therefore been designed to isolate individual features (for example single pulses) and interaction mechanisms in order to study them quantitatively. Since 2001 we have been able to dynamically change the surface catalytic activity in real time and space by focusing an addressable laser beam to differentially heat a $\mathrm{Pt}(110)$ single crystal surface. The combination between the fixed microstructures of metals with different catalytic activities and local laser heating of the surface has been recently explored.
\end{abstract}

KEYWORDS : CO-oxidation, imaging of surface reactions, chaos control, pattern formation

Non-linear phenomena during simple surface reactions became apparent early in the 1970s. By measuring the reaction rate during $\mathrm{CO}$-oxidation in an open system at atmospheric pressure by passing the reactants over a heated Platinum wire, the Wicke group in Münster, Germany, observed rate oscillation for the first time ${ }^{1)}$. In about 1980 Gerhard Ertl, at that time at the Ludwig Maximilians University in Munich, embarked on a mission to tackle these astounding findings with a compilation of powerful surface science tools. Soon his group was able to explain many of the phenomena, establishing a nonlinear surface reaction as a paradigm for non-linear dynamics $^{2 \sim 5)}$. The question about local interdependencies on oscillations remained mostly unsolved. After Gerhard Ertl moved to the Fritz-Haber-Institute of the Max/Planck Society in 1986 as Director of the Department for Physical Chemistry, he asked me to form a new research group dedicated to the imaging of surface reactions. We were determined to find any spatial involvement in the temporal oscillations.

The first step was to build a scanning photoemission microscope by focusing a UV-light source to a $0.5 \mu \mathrm{m}$ spot and measuring the total yield of emitted electrons for each spot on the surface via a channeltron. By scanning the photon beam across the

E-mail : harm.rotermund@dal.ca surface we obtained an image of the local work function of the surface ${ }^{6)}$. This new method was successfully applied to the CO-oxidation on $\mathrm{Pt}(100)$, where the oscillations where in the minute range ${ }^{7)}$. It became immediately apparent to us that we would need a real time imaging technique to succeed.

We decided to construct a simple ultra high vacuum (UHV) compatible photoemission electron microscope (PEEM). The PEEM yields spatially resolved real-time images of the adsorbate-dependent local work function that can be readily translated into coverage patterns. Depending on the difference in work function between $\mathrm{CO}$-and $\mathrm{O}$-covered $\mathrm{Pt}, \mathrm{CO}-$ covered areas appear bright in the PEEM image, whereas O-covered regions are dark. The PEEM is sketched in Fig. 1 illustrating the sample with high electron emission from $\mathrm{CO}$ regions versus low emission from oxygen-covered areas. The light source for emitting electrons is a Deuterium discharge lamp delivering photons up to $6.8 \mathrm{eV}$. The PEEM features an aperture in the first focal point of the objective lens, allowing differential pumping so that the reaction chamber pressure can be raised from UHV up to $10^{-3}$ mbar, while the imaging channelplate and the adjacent phosphorous screen remain below $10^{-6}$ mbar. The very first images from the PEEM, using just the cathode lens and no image intensifier, required to adapt to a completely dark laboratory and 


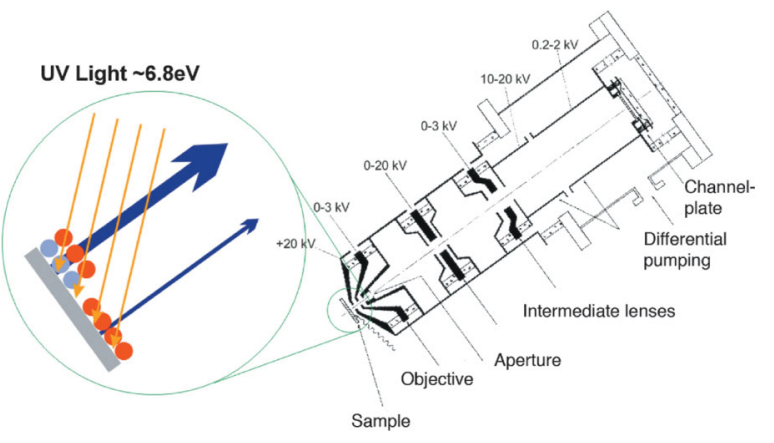

Fig. 1. (color online). Sketch up of our PEEM, consisting of electrostatic electron lenses, sample potential is near ground, typical magnification $100-1000 \times$, field of view up to $600 \mu \mathrm{m}$ diameter, spatial resolution 0.1 $\mu \mathrm{m}$, time resolution $40 \mathrm{~ms}$.

took Gerhard Ertl and me completely by surprise. They opened an avenue to an unseen world in twodimensional pattern formation ${ }^{8)}$. As soon as our workshop had completed the other parts for the PEEM and we finished the assembly of it, the whole complexity and beauty of pattern formation during CO-oxidation on $\operatorname{Pt}(110)$ became apparent ${ }^{9 \sim 14)}$. During the early years we also explored the potential for the new technique. These investigations included diffusion experiments for $\mathrm{CO}$ and $\mathrm{O}$, which allowed the simultaneous determination of coverage and directional dependencies ${ }^{15 \sim 17)}$.

To illustrate two different phenomena prevalent in pattern formation during surface reactions at low pressures, Fig. 2 presents snapshots of target patterns during the CO-oxidation on $\operatorname{Pt}(110)$, reproduced from ${ }^{18)}$. This figure shows the evolution of various target patterns in addition to a periodically changing background. The latter is a clear indication for a globally coupled system, via gas phase coupling, while the targets are dominated by diffusion coupling.

Coupling does not occur between the target patterns themselves or between them and the global background oscillations ${ }^{18)}$. This behavior is typical for patterns originating from a reaction diffusion system. The faster (compared to the characteristic reaction time) information between neighboring parts of the catalyst is exchanged, the larger the characteristic length. In heterogeneous reactions at low pressures, the dominant spatial coupling (transport) is diffusion of at least one of the adsorbates like $\mathrm{CO}$, with a typical diffusion length on the order of $\mu \mathrm{m}$. This can be estimated from the square root of the diffusion coefficient for $\mathrm{CO}$ multiplied by a typical time until a desorption or reaction process will happen. Obviously the target patterns are more robust than the oscillating
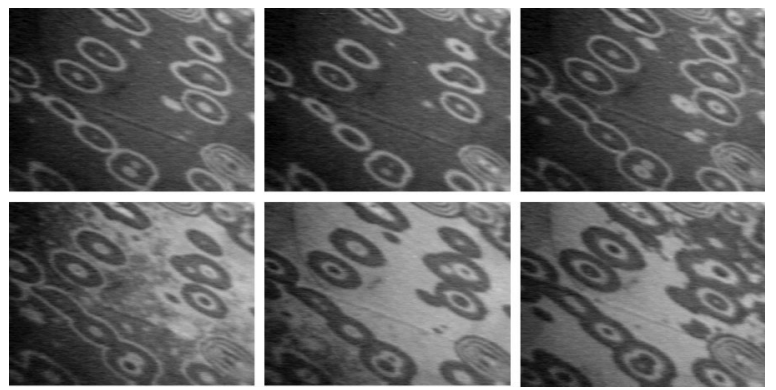

Fig. 2. Snapshots of target pattern, $\mathrm{p}_{\mathrm{O}_{2}}=3.2 \times 10^{-4}$ mbar, $\mathrm{p}_{\mathrm{CO}}=3 \times 10^{-5} \mathrm{mbar}$ and $\mathrm{T}=427 \mathrm{~K}$, time interval $1 \mathrm{~s}$, frame size $300 \times 227 \mu \mathrm{m}$.
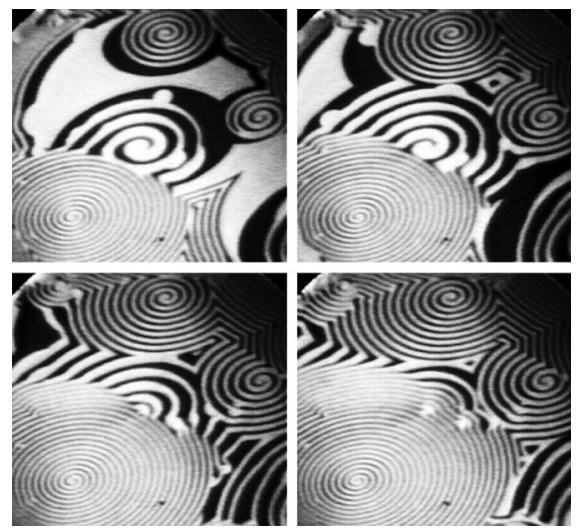

Fig. 3. Evolving spirals, $\mathrm{T}=448 \mathrm{~K}, \mathrm{p}_{\mathrm{o}_{2}}=4 \times 10^{-4} \mathrm{mbar}$, $\mathrm{p}_{\mathrm{CO}}=4.3 \times 10^{-5} \mathrm{mbar}$, time between frames $30 \mathrm{~s}$, frame size $440 \times 440 \mu \mathrm{m}$.

background and Fig. 2 seems to have captured a situation where the system has been at a transient between an oscillatory and an excited state. When the reaction parameters are chosen so that the system is in its excited state, evolving spirals are the most dominant features. Figure 3 shows a beautiful example of a set of left and right rotating spirals with different wavelengths ${ }^{9}$. The spirals are all pinned with their core to macroscopic defects on the $\mathrm{Pt}(110)$ surface and depending on the size of those defects their rotation speed, and therefore their wavelength, varies. Note that the rotation direction is arbitrary dependent upon which side of the initial wave encounters the defect.

It became obvious that various forms of patterns may interfere at the same time and it would be advantageous to isolate and control pattern formation. In principle two approaches to steering patterns are feasible : local control by confining pulses in fixed deposited structures on the catalytic surface, or by writing temperature patterns as boundaries for the pulses in real time. The other approach utilizes global 
control via direct forcing (modulating the gas phase), via global delayed feedback, or more complex nonuniform coupling schemes. Various methods have been proven to suppress chaos or turbulence, one of the challenging problems in nonlinear dynamics.

In 1993 we started to study boundary conditions for pattern formation. Evaporating sub-monolayer amounts of Au through a mask onto a single crystal of Platinum was the first step in controlling non-linear phenomena during surface reactions ${ }^{19,20)}$. If patches with different composition (such as bare and Aucovered $\mathrm{Pt}(110)$ ) are adjacent, waves from the excitable region may penetrate into the mono-stable area before they are extinguished. This behavior is beautifully illustrated in Fig. 4, reproduced from ${ }^{20)}$. There an O-spiral with its core on the Au-covered part is emitting waves into the bare Pt area, where their width increases considerably. The speed along the fast direction increases from $0.8 \pm 0.2 \mu \mathrm{m} / \mathrm{s}$ to $1.3 \pm 0.2$ $\mu \mathrm{m} / \mathrm{s}$. The wavelength of the spirals on the bare $\mathrm{Pt}$ area is therefore larger than on the $\mathrm{Au}$ covered surface. This was the first example showing refraction of chemical reaction waves, obeying Snellius' law for optical waves. The Au-covered Pt(100) surface showed similar behavior, although no total reflection could be observed $^{21)}$. The investigations were done by in-situ evaporation of $\mathrm{Au}$ atoms.

These experiments were restricted to comparatively wide structures of simple geometry due to the kind of mask, which could be used. Using microlithography, which is inherently an ex-situ method, allowed for much finer boundaries with an open-end variety of geometric arrangements. Depending on the material used as boundaries we can distinguish between catalytic non-active ${ }^{22 \sim 24)}$, versus active boundary conditions ${ }^{25,26)}$. In Ref. 26 we show an example where pattern formation depends on the catalyst composites, and in particular, the effect of the composite on the shape and interaction of pulses on the $\operatorname{Pt}(110)$ part of the composite. Pulses constitute the main building blocks of more complex patterns, such as targets and

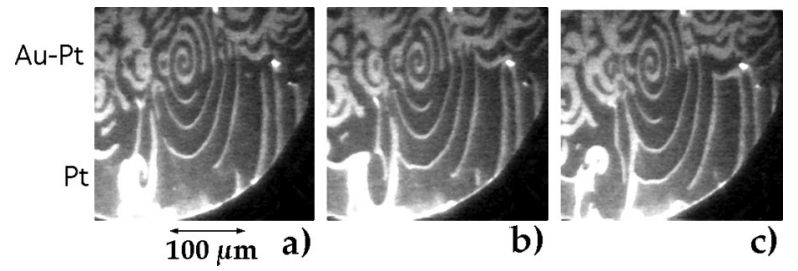

Fig. 4. Periodic wave trains originating from a spiral centered on the Au-covered part. $\mathrm{T}=480 \mathrm{~K}, \mathrm{p}_{\mathrm{O}_{2}}=$ $4 \times 10^{-4}$ mbar, $\mathrm{p}_{\mathrm{CO}}=4.8 \times 10^{-5}$ mbar. spirals. This "perturbation" to the pattern forming dynamics on the Pt can be tailored through the choice of a companion component, as well as through the geometry of the composite (composite composition, stripe width). Figure 5 is reproduced from Ref. 26 to illustrate this kind of interaction between the boundaries and reaction pulses. The standard pulse geometry, which is highly symmetrical, becomes drastically changed. The triangular shaped pulses move forward with their broad side. We found that the qualitative effect on the shape and interaction of the pulses (the introduction of distinct two-dimensional features based on the front-back asymmetry of the pulse) was well captured theoretically through a mechanistic model of $\mathrm{CO}$ oxidation $^{27)}$.

These structures are fixed and cannot be altered after they have been designed. The development of new optical imaging techniques, ellipsomicroscopy for surface imaging (EMSI) and reflection anisotropy microscopy (RAM $)^{28)}$, not only allow the observation of pattern formation at any pressure, but also enable the in-situ creation of temperature heterogeneities as boundaries by focusing a laser beam onto the

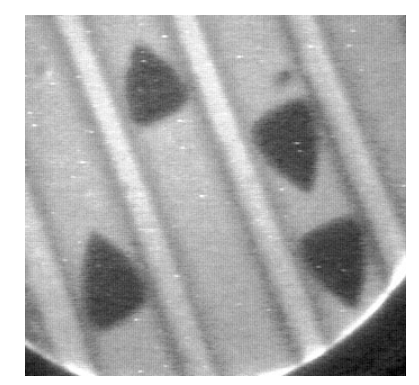

Fig. 5. Experimental PEEM image of $\mathrm{O}$ pulses in $22 \mu \mathrm{m}$ wide $\mathrm{Pt}$ channels on a $\mathrm{Rh} / \mathrm{Pt}$ surface $\mathrm{T}=440 \mathrm{~K}$, $\mathrm{p}_{\mathrm{O}_{2}}=4.3 \times 10^{-4}$ mbar, $\mathrm{p}_{\mathrm{CO}}=3.2 \times 10^{-5} \mathrm{mbar}$.

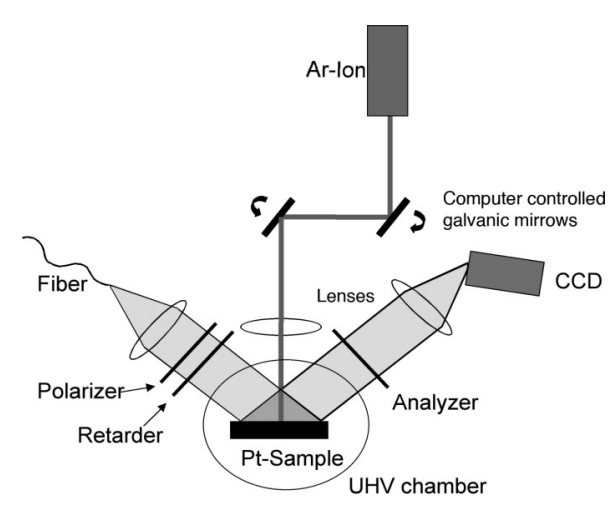

Fig. 6. Set up of ellipsomicroscopy for surface imaging (EMSI) including local heating of the surface by an addressable focused laser spot. 
(a)

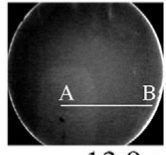

$13.9 \mathrm{~s}$
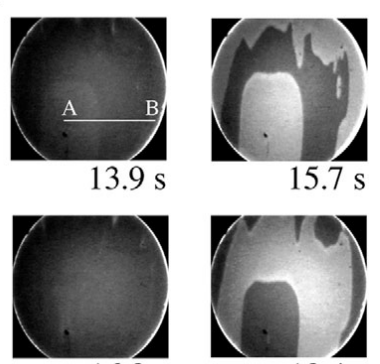

$16.8 \mathrm{~s}$

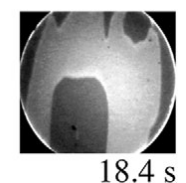

(b)

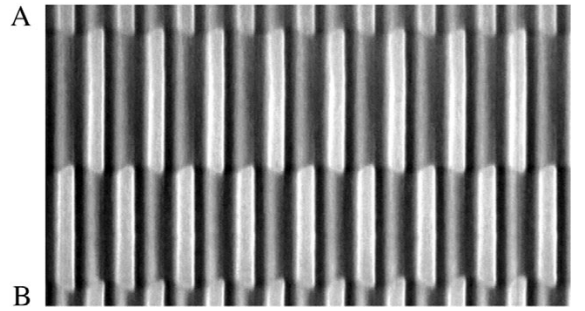

Fig. 7. Oscillatory cluster pattern, $\mathrm{T}=506 \mathrm{~K}, \mathrm{p}_{\mathrm{O}_{2}}=4 \times 10^{-4}$ mbar, $\mathrm{p}_{\mathrm{CO}}=2.2 \times 10^{-5} \mathrm{mbar}, \partial \mathrm{t}$ (the time delay) $=$ $0.45 \mathrm{~s}, \mu$ (the intensity of the time delayed feedback signal) $=3.5 \times 10^{-5}$ mbar, image diameter $350 \mu \mathrm{m}$, a) Typical PEEM images during one period. b) Space/ time plot along line AB indicated in the first PEEM image.

surface $^{29)}$. This new technique is illustrated in Fig. 6, where a monochromatic light beam from a laser is elliptically polarized by a polarizer and a retarder (a quarter wave plate), such that the beam after reflection from the sample is linearly polarized. This allows us to analyze the reflected light with another polarizer (analyzer) and image the beam directly onto a CCD camera. Any difference in the adsobate coverage, i.e. islands of oxygen atoms on a $\mathrm{CO}$ covered $\mathrm{Pt}$ surface, will result in contrast for the image. The contrast arises from a different dielectric constant for $\mathrm{CO}$ molecules versus oxygen atoms and/or their respective heights. Areas need to be above the optical resolution to become visible in an image ; in our set up we are able to see regions with different adsobates as small as $30 \mu \mathrm{m}^{2}$. A recent improvement of RAM resolution allowed us to combine fixed composite catalytic structures with laser beam actuation of pulses $^{30)}$. EMSI has not been capable of looking at pattern formation inside of microlithography structures, since those structures are orders of magnitude taller than the adsorbate patterns themselves, therefore the intensity originating from the boundaries would overwhelm any other signal.

So far the interaction between the reaction diffusion system and the outside control function has been utilized by locally changing the diffusivity of the reactants on the catalytic surface. As it was already indicated in Fig. 2, a different information exchange during oscillating surface reactions occur via global coupling through the gas phase supplying the reactants. This opens a new strategy for control. Encouraged by Alexander Mikhailov and his theoretical studies suggesting a method to control turbulence by applying an appropriate time delayed global feedback $^{31,32)}$, we set up an experiment in which we used the integrated PEEM intensity in a delayed feedback loop to suppress the previously established turbulent state ${ }^{33 \sim 35)}$. As an example, Fig. 7(a), taken from $^{34)}$, shows typical PEEM snapshots after decreasing the intensity of the feedback from high values, where homogeneous oscillations are found, to lower values. These cluster patterns have not been observed without feedback. Figure 7(b) presents a space/time plot along the line $\mathrm{AB}$ shown in the first image of Fig. 7 (a), showing that the system has nearly reached a state of phase balance.

Although the CO-oxidation is a simple, twodimensional, reaction diffusion system, it proved again to be a paradigm for non-linear dynamics. This experimental verification of "chaos control" in turn inspired new theoretical work, "Taming the Winfree turbulence of scroll waves" ${ }^{36)}$, which is an important step for understanding and suppressing cardiac fibrillation.

Recently we explored the simplest approach to control by direct forcing. This is achieved by imposing an oscillation onto one of the feeding lines, which is then passed through the dosing valve into the reaction chamber. A common problem is the slow response when trying to electronically impress fast changes to the feeding line. By attaching a mechanical plunger to the feeding line, which could be rapidly moved in and out by a stepping motor, we could more than double the forcing frequency ${ }^{37)}$. This allowed period resonant forcing up to $4: 1$ of the natural frequency, a parameter region not previously accessible $^{38)}$.

For 20 years we explored the unknown of reaction diffusion systems occurring in surface reactions. Throughout the years Gerhard Ertl maintained a deep curiosity into their nature and he continues to be an enthusiastic supporter, an inspiring collaborator and a friend.

\section{References}

1) E. Wicke, P. Kumann, W. Keil and J. Schiefler : Ber. Bunsenges. Phys. Chem. 84, 315 (1980). 
2) M.P. Cox, G. Ertl and R. Imbihl : Phys. Rev. Lett. 54, 1725 (1985).

3) M.P. Cox, G. Ertl, R. Imbihl and J. Rüstig : Surf. Sci. 134, L517 (1983).

4) P.A. Thiel, R.J. Behm, P.R. Norton and G. Ertl : Surf. Sci. 121, L553 (1982).

5) G. Ertl, P.R. Norton and J. Rüstig : Phys. Rev. Lett. 49, 177 (1982).

6) H.H. Rotermund, G. Ertl and W. Sesselmann : Surf. Sci. 217, L383 (1989).

7) H.H. Rotermund, S. Jakubith, A.v. Oertzen and G. Ertl : J. Chem. Phys. 91, 4942 (1989).

8) H.H. Rotermund, W. Engel, M. Kordesch and G. Ertl : Nature 343, 355 (1990).

9) S. Nettesheim, A.v. Oertzen, H.H. Rotermund and G. Ertl : J. Chem. Phys. 98, 9977 (1993).

10) M. Bär, M. Eiswirth, H.H. Rotermund and G. Ertl : Phys. Rev. Lett. 69, 945 (1992).

11) H.H. Rotermund, S. Jakubith, A.v. Oertzen and G. Ertl : Phys. Rev. Lett. 66, 3083 (1991).

12) S. Jakubith, H.H. Rotermund, W. Engel, A.v. Oertzen and G. Ertl : Phys. Rev. Lett. 65, 3013 (1990).

13) M. Bär, S. Nettesheim, H.H. Rotermund, M. Eiswirth and G. Ertl : Phys. Rev. Lett. 74, 1246 (1995).

14) J. Lauterbach and H.H. Rotermund : Surf. Sci. 311, 231 (1994).

15) A.v. Oertzen, H.H. Rotermund and S. Nettesheim : Chem. Phys. Lett. 199, 131 (1992).

16) H.H. Rotermund, S. Nettesheim, A.v. Oertzen and G. Ertl : Surf. Sci. 275, L645 (1992).

17) A. von Oertzen, H.H. Rotermund and G. Ertl : Chem. Phys. Lett. 199, 131 (1992).

18) H.H. Rotermund: "Pattern Formation in a Surface Reaction with Global Coupling” (Springer, Minneapolis, 1999) p. 231.

19) K. Asakura, J. Lauterbach, H.H. Rotermund and G. Ertl : Phys. Rev. B 50, 8043 (1994).

20) K. Asakura, J. Lauterbach, H.H. Rotermund and G. Ertl : J. Chem. Phys. 102, 8175 (1995).

21) K. Asakura, J. Lauterbach, H.H. Rotermund and G. Ertl :
Surf. Sci. 374, 125 (1997).

22) M. D. Graham, I. G. Kevrekidis, K. Asakura, J. Lauterbach, K. Krischer, H.H. Rotermund and G. Ertl : Science 264, 80 (1994).

23) M.D. Graham, M. Bär, I.G. Kevrekidis, K. Asakura, J. Lauterbach, H.H. Rotermund and G. Ertl : Phys. Rev. E 52, 76 (1995).

24) G. Haas, M. Bär, I.G. Kevrekidis, P.B. Rasmussen, H.H. Rotermund and G. Ertl : Phys. Rev. Lett. 75, 3560 (1995).

25) J. Lauterbach, K. Asakura, P.B. Rasmussen, H.H. Rotermund, M. Bär, M.D. Graham, I.G. Kevrekidis and G. Ertl : Physica D 123, 493 (1998).

26) M. Pollmann, H.H. Rotermund, G. Ertl, X. Li and I.G. Kevrekidis : Phys. Rev. Lett. 86, 6038 (2001).

27) X. Li, I.G. Kevrekidis, M. Pollmann, A.G. Papathanasiou and H.H. Rotermund: Chaos 12, 190 (2002).

28) H.H. Rotermund, G. Haas, R.U. Franz, R.M. Tromp and G. Ertl : Science 270, 608 (1995).

29) J. Wolff, A.G. Papathanasiou, I.G. Kevrekidis, H.H. Rotermund and G. Ertl : Science 294, 134 (2001).

30) C. Punckt, F.S. Merkt and H.H. Rotermund : New Journal of Physics 9, 213 (2007).

31) D. Battogtokh and A. Mikhailov: Physica D 90, 84 (1996)

32) D. Battogtokh, A. Preusser and A. Mikhailov: Physica D 106, 327 (1997).

33) M. Kim, M. Bertram, M. Pollmann, A. von Oertzen, A.S. Mikhailov, H.H. Rotermund and G. Ertl : Science 292, 1357 (2001).

34) M. Pollmann, M. Bertram and H.H. Rotermund : Chem. Phys. Lett. 346, 123 (2001).

35) M. Bertram, C. Beta, M. Pollmann, A.S. Mikhailov, H.H. Rotermund and G. Ertl : Phys. Rev. E 67, 036208 (2003).

36) S. Alonso, F. Sagues and A.S. Mikhailov : Science 299, 1722 (2003).

37) P.S. Bodega, P. Kaira, C. Beta, D. Krefting, D. Bauer, B. Mirwald-Schulz, C. Punckt and H.H. Rotermund : New Journal of Physics 9, 61 (2007).

38) P. Kaira, P.S. Bodega, C. Punckt, H.H. Rotermund and D. Krefting : Phys. Rev. E, in press (2008). 